\title{
全球变暖与陆地生态系统研究中的野外增温装置
}

\author{
牛书丽 韩兴国 马克平 万师强* \\ (中国科学院植物研究所植被数量生态学重点实验室, 北京 100093)
}

\begin{abstract}
摘 要 由于化石燃料燃烧和森林砍伐等人类活动引起的地球大气层中温室气体(主要是二氧化碳)的富集已导 致全球平均温度在 20 世纪升高了 $0.6{ }^{\circ} \mathrm{C}$, 并将在本世纪继续上升 $1.4 \sim 5.8{ }^{\circ} \mathrm{C}$ 。这种地质历史上前所未有的全球 变暖将对陆地植物和生态系统产生深远影响, 并通过全球碳循环的改变反馈于全球气候变化。作为全球变化生态 学的主要研究方法之一,生态系统增温实验能够为生态模型提供参数估计和模型验证。然而由于在世界各地使用 的增温装置不同, 使得各个生态系统之间的结果比较和整合难以实施 增加了模型预测的不确定性。该文通过比 较几种常见的野外增温装置在模拟全球变暖情形时的优缺点, 指出利用不同增温装置进行全球变暖研究中应注意 的一些问题; 同时探讨了全球变暖控制实验研究中的一些关键性的科学问题。
\end{abstract}

关键词 全球变暖 增温装置 植物 生态系统 红外线辐射器

\section{FIELD FACILITIES IN GLOBAL WARMING AND TERRESTRIAL ECOSYSTEM RE- SEARCH}

\author{
NIU Shu-Li , HAN Xing-Guo , MA Ke-Ping , and WAN Shi-Qiang * \\ Laboratory of Quantitative Vegetation Ecology , Institute of Botany, Chinese Academy of Sciences , Beijing , 100093 , China
}

\begin{abstract}
Enrichment of atmospheric greenhouse gases resulted from human activities such as fossil fuel burning and deforestation has increased global mean temperature by $0.6{ }^{\circ} \mathrm{C}$ in the 20 th century and is predicted to increase it by $1.4-5.8{ }^{\circ} \mathrm{C}$ in this century. The unprecedented global warming will have profound long-term impacts on terrestrial plants and ecosystems. Responses of terrestrial plants and ecosystems to global warming may feed back to climate change via ecosystem and global carbon cycling. As one of the major methodology in global change research, ecosystem warming studies can facilitate model projections on potential changes in terrestrial biomes in terms of parameterization and validation. The difficulty in comparing and integrating the results from various ecosystems manipulated with different warming facilities exacerbated the uncertainties of model prediction. In this paper, field facilities in simulating climate warming and their potential applications in different terrestrial biomes were discussed. Critical scientific questions that could be addressed by ecosystem warming studies were proposed.
\end{abstract}

Key words global warming, warming facilities , plant, ecosystem , infrared radiator

自工业化革命以来，人类活动包括化石燃料的 燃烧和土地利用/覆盖度的变化已使地球大气层中 $\mathrm{CO}_{2}$ 的浓度上升了 $30 \%$, 其结果造成地球表面的平 均温度在 20 世纪升高了 $0.60{ }^{\circ} \mathrm{C}$, 预计到本世纪末 地球的平均温度还将继续上升 $1.4 \sim 5.8{ }^{\circ} \mathrm{C}$ ( IPCC, 2001 )。由于所有的物理、化学和生物学过程都对温 度反应敏感, 上述地质历史上前所未有的气候变化 将对陆地植物和动物的生长和分布以及生态系统的 结构和功能产生深远的影响, 并通过生态系统和全 球碳循环反馈于全球气候变化。

野外自然条件下的生态系统增温实验是研究全
球变暖与陆地生态系统关系的主要方法之一 ( Shen \& Harte, 2000) , 其研究结果为陆地生态系统结构与 功能的中长期动态模型预测和验证提供关键的参数 估计。为了有效地整合和总结世界各地开展的生态 系统增温实验, IGBP(International Geosphere and Biosphere Programme) 的核心项目 GCTE ( Global Change and Terrestrial Ecosystems) 成立了生态系统增温研究 网络 (NEWS : Network of Ecosystem Warming Studies)。 在该网络中广泛用于各种生态系统类型的温度控制 装置可以分为 4 大类 :1) 温室 (Greenhouse) 和开顶箱 (Open-top chamber)，2）土壤加热管道和电缆（Soil 
heating pipes and cables) 3)红外线反射器 (Infrared reflector) A) 红外线辐射器 (Infrared radiator)。由于这 些增温装置在设计、技术和增温机制上的差别，实验 增温后陆地植物和生态系统的响应各不相同。比较 这些增温装置之间模拟全球变暖机制和温度变化情 形(Temperature scenario)的有效性，有利于对陆地生 态系统的响应与适应得出全面和令人信服的结论。

全球变暖是由大气层中温室气体反射的长波辐 射(主要是红外线)增强而引起的。这种增强的向下 红外线辐射以 3 种能量形式分配:显热 (Sensible heat)、土壤热通量 (Soil heat flux) 和潜热 (Latent heat)。显热增加的结果是空气温度升高, 土壤热通 量增加导致土壤温度升高, 潜热增强引起蒸发散增 加。全球变暖背景下温度的变化有 3 种情形 :1) 夜 间增温幅度大于白天, 导致温度日变幅的降低，2)冬 季增温幅度大于夏季 ; $)$ 高纬度地区增温幅度大于 低纬度地区 ( IPCC, 2001)。因此，一种增温装置在 模拟全球变暖时是否有效, 需要判断其能否较好地 模拟全球变暖的机制 (增强的向下红外线辐射)、能 否同时改变气温、土壤温度和蒸发散以及能否模拟 上述几种增温情形。

本文将分别介绍上述几种增温装置并比较其优 缺点和增温效果。

\section{1 几种增温装置}

1.1 温室 (Greenhouse) 和开顶箱 (Open-top chamber , OTC)

最简单和最普遍的一种增温方法就是被动增温 的温室或开顶箱 (Richardson et al .,2000) , 这种增温 设施已经在一系列的生境中被应用, 主要是一些高 纬度和高海拔地区 (Chapin \& Shaver , 1985 ; Havström et al. , 1993 ; Norby et al., 1997 ; Oechel et al. , 1998 ; Hollister \& Webber , 2000 ; Klein et al . , 2005 ; Walker et al. ,2006)，包括北极和南极冻原、亚高山 草地、青藏高原和温带草原 (图 1)。温室和开顶箱 不仅可以用于增温处理, 而且还可以应用于其它气 候变化, 例如水分 (土壤湿度)、光照 (强度和光质)和 $\mathrm{CO}_{2}$ 等的研究当中。温室和开顶箱一般来说可以增 加空气温度 $2 \sim 6{ }^{\circ} \mathrm{C}$ (Stenstrom et al ., 1997 ; Klein et $a l ., 2005)$, 具体的温度要根据实验的目的和实际 情况而定。温室和开顶箱根据研究目的不同有很多 种材料和样式, 包括开顶式设计、园艺用钟形玻璃 盖、圆顶式帐篷、屏风式、玻璃温室、塑料温室以及纤 维板等。对于材料和结构式样的选择同等重要, 因
为不同材料的温室对于温度的升高幅度、光的衰减 程度以及气体的透过率都是不同的。用温室和开顶 箱作增温实验时,一般用同样材料制作的遮光板作 为对照来模拟遮光效应 (Debevec \& Maclean , 1993)。

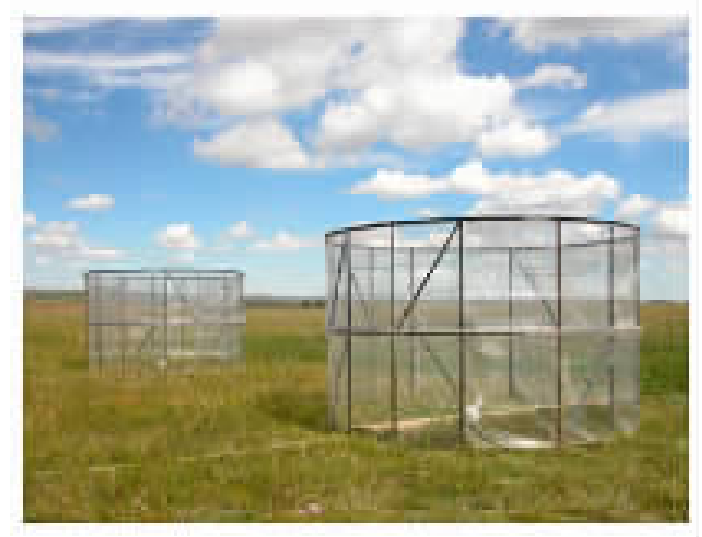

图 1 用于我国北方温带草原的开顶箱(照片由万师强提供)

Fig. 1 Open-top chambers used in a temperate steppe in northern China (Photo from Shiqiang Wan)

温室和开顶箱是最经济、简单易行的增温装置， 维持费用也不高, 可以用于一些偏远没有电力的地 区。例如 Chapin 等 (1995) 用温室在北极冻原地带 进行了连续 9 年的实验。尽管温室和开顶箱有很多 优点并且被广泛应用于气候变化的研究中, 这种被 动的增温方式也有很多缺点。在有些生态系统尤其 是在北极、南极和高山地区，由于冬季恶劣的天气， 用温室和开顶箱进行增温的可行性很低。冬季被认 为是增温幅度最大的一个季节 (IPCC，2001), 这对 于量化碳的年际收支和其它生物化学元素是很关键 的。人们争论过温室和开顶箱可能对于气候变化的 模拟并不充分 (Kennedy，1995) ,并且温度升高的幅 度和特征不能被很好地控制。温室不仅影响温度， 还影响到湿度、气体组成、雪的覆盖度 (由此产生的 生长季节的变化) 、光照 (强度和光质) 以及风速等。 温室和开顶箱还可以阻挡雨雪、降低混合气体的扩 散和湍流，这样就抑制了白天水蒸气的向上运动和 晚上露水的形成。这些负面效应增加了观察生态系 统对温度升高反应的难度, 使我们不能全面而真实 地理解全球变暖条件下微气候变化的综合效应。另 外, 温室或开顶箱还通过改变风速和阻挡动物活动 而影响植物花粉/种子的传播和有性生殖。

1.2 土壤加热管道和电缆 (Heating fluid pipes and electric resistance cables)

陆地生态系统包括荒漠、草地、灌丛和森林等各 种类型, 温室和开顶箱虽然能在荒漠、草地和灌丛等 
灌层较低的生态系统得到很好的应用, 但对于高灌 层的森林生态系统其应用被限制, 而且很难设置重 复。因此一种能在森林生态系统中得到应用的增温 方式随后得以问世, 这就是在土壤中掩埋电缆, 这种 增温方式的问世得益于土壤加热管道的启示。土壤 加热管道早在 20 世纪 70 年代就被用于农业实验研 究中, 俄勒冈州立大学的 Rykbost 等 (1975)利用发电 厂的废热水, 通过埋在地下 $92 \mathrm{~cm}$ 深处的管道对作 物和蔬菜进行增温处理。他们的目的不是模拟气候 变暖, 而是想探讨利用掩埋加热管道所产生的热量 来刺激植物生长的可能性, 显然, 这种方法可以应用 于研究气候变暖。运用土壤加热电缆研究全球变暖 效应的报道始见于 20 世纪 90 年代。van Cleve 等 (1983，1990) 用此法在阿拉斯加对森林生态系统进 行了大约 $9{ }^{\circ} \mathrm{C}$ 的土壤增温实验;Peterjohn 等 (1993, 1994) 设计和应用了此系统在北美洲温带落叶森林 里对土壤进行加热, 他们利用自动调温器从而保持 稳定的比对照高出 $5{ }^{\circ} \mathrm{C}$ 的土壤温度。Peterjohn 等 (1993)建议用此装置进行增温时应该有两种对照： 一种是在相同深度的土壤层埋上电缆但不加热, 模 拟由于埋线所造成的干扰; 另一种是纯粹的对照， 即不埋设电缆。埋地电缆可以通过适宜的电路控制 从而得到一个稳定的可控的温度, 它不像温室那样 引起微气候环境的改变。尽管这种装置需要电力， 在没有电力设施的地方受到限制, 然而它是目前研 究全球变暖对于森林生态系统影响的可行的手段。 但是, 运用埋地电缆对土壤进行增温有以下缺点: 1) 在掩埋电缆时对土壤和地表枯枝落叶层造成物理 干扰，尽管 Peterjohn 等(1993) 发现这种干扰对日均 温并没有影响, 但其它土壤和生态系统过程, 例如气 体的扩散、水分的径流、中型动物的活动以及根系等 都有可能受到影响 (NSFESP，1991），2)加热不均匀， 埋地电缆会在土壤中造成垂直和水平的温度梯度 ; 3)埋地电缆不能加热空气和植物地上部分, 因而不 能比较真实地模拟全球变暖影响陆地生态系统的情 形 ;)土壤管道和电缆所造成的恒定增温不能模拟 自然条件下全球变暖所引起的增温幅度的季节和日 间变化。

一种和埋地电缆原理相似但同时能在低灌层生 态系统得以应用的土壤加热方法就是在土壤表面进 行管道加热。Hiller 等 (1994) 用一种不锈钢管充满 液体, 置于土壤的表层制造一种连续的稳定的温度 差异。他们利用这种管道系统在谢菲尔德的一个花 园里控制生长季节温度, 从而模拟温暖的冬季。每
一个加热的小区对应一个布置了不加热管道系统的 对照小区，以便消除装置的遮荫效果。这种管道系 统被一个金属栅格所支撑, 这种金属栅格还可以侧 面散热，从而创造一种相对均匀分布的温度条件。 与掩埋电缆相比, 这种增温方法对土壤和植被造成 的物理干扰较小, 它也不像温室和开顶箱那样阻断 雨雪、降低风速和削弱光照。这种设置既可以用于 增温也可以用于降温, 对于裸露的土壤或低植被层 的生态系统无疑是一种控制土壤温度的好方法。

\section{3 红外线反射器 (Infrared reflectors)}

随着对全球变暖情形的深入了解, 人类认识到 全球变暖背景下夜间的增温幅度大于白天的增温幅 度 (IPCC，2001) ,因此，一种比较好的模拟夜间增温 的实验装置一红外线反射器得以问世和应用 (Zeiher et al ., 1994 ; Luxmoore et al , , 1998 ; Beier et al. , 2004 ; Emmett et al. , 2004)。它的原理和设计 也比较简单，加热小区周围的支架可以收放反射红 外线的穷布, 这种穷布可以反射 $97 \%$ 的直射光和 $96 \%$ 的散射光, 同时允许水蒸气透过。穷布可以被 卷进脚手架一端的一个横梁内, 此横梁与一个马达 相连以供应电力。马达被一个电子控制器控制自动 开动, 帘布可以自动伸卷, 整个伸卷过程大约需要 4 $\min$ 。在每天日落的时候 (光密度大约小于 $0.4 \mathrm{~W}$ $\mathrm{m}^{-2}$ ), 帘布可以自动地伸开并覆盖植被以降低红外 线辐射所造成的能量散失, 在早晨太阳升起时, 穷布 被自动卷回，从而使加热的实验小区保持白天的开 放状态。另外此装置还可以连接雨水感应器和风速 感应器, 在有雨或大风的夜晚也会自动卷起穷布 (Beier et al. , 2004)。

这种装置可以成功地将年均最低气温和土温增 加 $0.4 \sim 1.2{ }^{\circ} \mathrm{C}$, 而且加热设置的边际效应相对较小 (Beier et al.,2004)。该装置相对经济易行，已经被 成功地应用于农作物和灌丛生态系统。但是在实际 操作过程中，一些潜在的负面效应还是要注意的，尤 其是在干旱地区，清晨露水的输入对于水分的收支 很重要, 该装置降低了露水的输入; 另外该装置没有 降雪感应器, 阻止了加热小区内雨雪的降落; 可能还 有其它一些非生物因素, 比如在有风的夜晚降低风 速等对实验所造成的影响。

\section{4 红外线辐射器 (Infrared radiators)}

为了真实地模拟全球变暖的机制，即增强的向 下红外线辐射，一种新的增温装置一红外线辐射 器在生态系统控制实验中逐渐得到应用。该装置是 通过悬挂在样地上方、可以散发红外线辐射的灯管 
来实现的(Shaver et al ，2000)(图 2)。

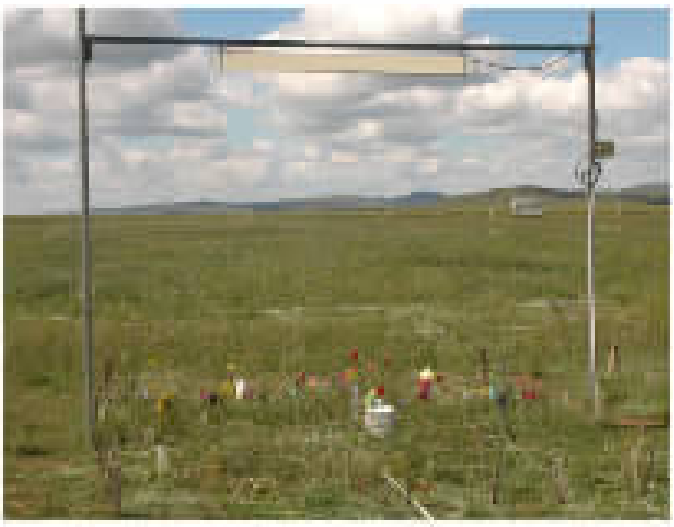

图 2 用于我国北方温带草原的红外线辐射器(照片由万师强提供) Fig.2 Infrared radiator used in a temperate steppe in northern China (Photo from Shiqiang Wan)

Harte 等 (1995) 首先应用红外线辐射器在美国 科罗拉多州亚高山草甸进行生态系统增温实验; Bridgham 等(1995)在明尼苏达州利用相同的装置研 究全球变暖对湿地生态系统的影响;Nijs 等 (1996) 也用红外线辐射器在瑞士对多年生黑麦草 (Lolium perenne)生态系统进行了加热实验; 另外, 这种增温 装置还先后被用于其它草地生态系统 ( Luo et al. , 2001 ; Shaw et al., 2002 ; Wan et al., 2002a ,b)。 Kimball (2005) 对红外线辐射器的加热效应和原理 进行了详细的论述, 并用理论公式模拟了利用红外 线辐射器对植物灌层进行加热时单位土地面积升高 单位温度时所需要的热辐射能量。

Nijs 等 (1996) 认为, 应用红外线辐射器从冠层 上面加热能够在植被层保持自然的温度梯度。红外 线灯管非破坏性地传递能量，而且不改变微环境，对 于那些冬季积雪比较厚的地方进行全年增温控制实 验也是可行的。但是由于辐射器并不直接加热空 气，这种技术不能模拟全球变暖的对流加热效应，而 且对于比较密集的植被层可能会削弱对土壤的增 温。另外, 由于该种加热装置所能覆盖的面积有限, 因此在森林生态系统中的应用受到限制。

\section{2 增温装置增温效果的比较}

以上提到的几种增温装置有着不同的增温效应 （表 1)，对于一天中温度的变化, 温室和开顶箱只能 在有太阳辐射的白天增加温度而降低夜间的气温 (Suzuki \& Kudo，1997；Werkman et al.，1996)，从而 可以使气温日较差增加 $1.2 \sim 1.8{ }^{\circ} \mathrm{C}$ 左右 (Marion et al ., 1997 ; Hollister \& Webber, 2000 ; Klein et al. , 2005)。对于季节间温度的变化而言, 温室和开顶箱 对于夏季的气温比冬季的气温增加的多 (Marion et al . 1997 ;Stenstrom et al . , 1997)。关于温室和开顶 箱控制下的土壤温度的变化, 前人的研究结果是不 一致的,有的认为没有变化 (Suzuki \& Kudo，1997; Hobbie et al ，1999) , 有的认为增加日均土温和日最 大土温但降低日最低土温（Marion et al.，1997； Robinson et al ., 1998 ; Shaver et al ., 1998 ; Day et $a l ., 1999)$ 。总的来说, 温室和开顶箱对气温的增加 比对土温高, 而且在有些研究中对于土壤湿度没有 影响。

土壤管道或电缆虽然能够保证加热样地的土壤 温度增加 $2.5 \sim 10{ }^{\circ} \mathrm{C}$ ( Peterjohn et al . , 1994) ,但是 这些装置在管道或电缆周围会引起土壤温度的梯度 变化 (Verberg et al . , 1999) ,并且很少影响到空气温 度, 除非它们与其它加热装置比如温室联合使用 (Hartley et al ，，1999）。单一土壤温度的增加只对植 物和生态系统的地下部分和过程产生较大的影响， 因而不能模拟在全球变暖条件下空气和土壤温度同 时增加的真实情形; 另外 相对恒定的增温不能真实 地模拟全球变暖条件下增温幅度的季节和日波动。

红外线反射器只能增加夜里温度和日最低气 温,它们通常对白天气温是没有影响的 (Zeiher et $a l$ ，，1994）这种温度变化的结果可能引起植物呼吸 的增加而对植物光合碳固定没有影响，从而影响了 对全球变暖条件下植物碳收支平衡的预测。据报 道 植物生长和生态系统的初级生产力在红外线反 射器处理的小区比对照小区要低 (Zeiher et al. , 1994 ; Luxmoore et al ，, 1998)。这与模型预测的全球 变暖条件下植物或生态系统的反应以及在多数生态 系统的实验观测结果 (Rustad et al. 2001; Wan et al.,2005)不一致。

红外线辐射器能够模拟全球变暖情形中增强的 向下的红外线辐射, 因而可以同时影响显热、潜热和 土壤热通量 (Harte et al., 1995; Wan et al. 2002a; Noormets et al., 2004 ; Kimball，2005)。然而,关于 红外线辐射器能否改变空气温度还没有一致的结 论。Nijs 等(1996)、Wan 等(2002a)和 Kimball (2005) 的实验结果表明, 红外线辐射器可以增加空气温 度, 但是 Harte 等 (1995) 认为红外线辐射器不能改变 空气温度。这可能与所研究的生态系统类型和其它 环境因子 (如风速) 有关。例如在风速较低、冠层高 而密的美国 Oklahoma 高草草原 红外线辐射器可以 
表 1 几种野外增温装置的比较

Table 1 The comparison among field warming facilities

\begin{tabular}{|c|c|c|c|}
\hline $\begin{array}{l}\text { 增温装置 } \\
\text { Warming facilities }\end{array}$ & 优点 Advantages & 缺点 Disadvantages & $\begin{array}{l}\text { 应用实例 } \\
\text { Implication instances }\end{array}$ \\
\hline $\begin{array}{l}\text { 温室/开顶箱 } \\
\text { Greenhouse/open-top chamber }\end{array}$ & $\begin{array}{l}\text { 简单易行 不需电力 经济 } \\
\text { Low cost in construction and mainte- } \\
\text { nance. No electric power needed }\end{array}$ & $\begin{array}{l}\text { 不能模拟全球变暖条件下增温 } \\
\text { 的日变化 影响小气候(光照、风 } \\
\text { 速、湿度和降雨)和动物活动 } \\
\text { Different diurnal patterns of temper- } \\
\text { ature change from those under natu- } \\
\text { ral condition. Disturbance to micro- } \\
\text { climate (light, wind speed, humidi- } \\
\text { ty and rainfall) and animal activities }\end{array}$ & $\begin{array}{l}\text { Chapin et al. , } 1995 \\
\text { Marion et al. , } 1997 \\
\text { Norby et al. , } 1997 \\
\text { Shaver et al. , } 1998 \\
\text { Klein et al . , } 2005\end{array}$ \\
\hline $\begin{array}{l}\text { 土壤加热管道和电缆 } \\
\text { Heating fluid pipes and electric } \\
\text { resistance cables }\end{array}$ & $\begin{array}{l}\text { 能精确的控制土壤温度 } \\
\text { Close control soil temperature }\end{array}$ & $\begin{array}{l}\text { 不能模拟全球变暖增温的季节 } \\
\text { 和日变化, 空间加热不均匀, 干 } \\
\text { 扰土壤, 影响土壤动物和微生物 } \\
\text { 的活动, 不能加热空气和植物地 } \\
\text { 上部分 } \\
\text { No temporal fluctuations of tempera- } \\
\text { ture increase. Spatial heterogeneity } \\
\text { of warming effect. Disturbance on } \\
\text { soil and soil fauna. No warming on } \\
\text { air temperature and aboveground } \\
\text { parts of plants }\end{array}$ & $\begin{array}{l}\text { van Cleve et al. , } 1990 \\
\text { Hiller et al. , } 1994 \\
\text { Peterjohn et al. , } 1994 \\
\text { Ineson et al . , } 1998 \\
\text { Bergh \& Linder , } 1999 \\
\text { Hartley et al . , } 1999\end{array}$ \\
\hline $\begin{array}{l}\text { 红外线反射器 } \\
\text { Infrared reflectors }\end{array}$ & $\begin{array}{l}\text { 能模拟全球变暖的增温机制和 } \\
\text { 日变化, 对土壤及植被无物理干 } \\
\text { 扰 } \\
\text { Better simulation of diurnal warming } \\
\text { patterns. No physical distrubance on } \\
\text { soil and plants }\end{array}$ & $\begin{array}{l}\text { 只能夜间增温 影响夜间小气候 } \\
\text { 和动物活动以及清晨露水的输 } \\
\text { 入 } \\
\text { No warming during daytime. Possi- } \\
\text { blely affect animal activities and dew } \\
\text { input }\end{array}$ & $\begin{array}{l}\text { Zeiher et al . , } 1994 \\
\text { Luxmoore et al. , } 1998 \\
\text { Beier et al . , } 2004 \\
\text { Emmett et al . , } 2004\end{array}$ \\
\hline $\begin{array}{l}\text { 红外线辐射器 } \\
\text { Infrared radiators }\end{array}$ & $\begin{array}{l}\text { 能模拟全球变暖的增温机制和 } \\
\text { 日变化, 对土壤及植被无物理干 } \\
\text { 扰,不改变小气候状况 } \\
\text { No disturbance on windspeed, soil } \\
\text { and plant }\end{array}$ & $\begin{array}{l}\text { 耗费电力较多,在没有电力的地 } \\
\text { 方和森林生态系统使用受到限 } \\
\text { 制 } \\
\text { Expensive in terms of energy cost. } \\
\text { Limited use in forest ecosystems and } \\
\text { area without electric power }\end{array}$ & $\begin{array}{l}\text { Harte et al. , } 1995 \\
\text { Bridgham et al. , } 1995 \\
\text { Luo } \text { et al . , 2001 } \\
\text { Wan et al. , 2002a } \\
\text { Noormets et al. , } 2004 \\
\text { Kimball , 2005 }\end{array}$ \\
\hline
\end{tabular}

使冠层日最高气温增加 $0.1{ }^{\circ} \mathrm{C}$, 而夜间最低温度增 加 $0.8 \sim 1.0{ }^{\circ} \mathrm{C}$, 从而降低了气温日较差 (Wan et $a l$. 2002a)。但是在风速较大、冠层低而稀疏的我 国北方温带典型草原, 红外线辐射器只增加土壤温 度而不改变空气温度(图 3)。

尽管不同类型的增温装置有着不同的增温效果 和缺点, 但是作为全球变化生态学研究的主要手段 之一, 生态系统增温实验在研究陆地生态系统对全 球变暖的响应和适应机理方面有着不可替代的作 用。例如, 加利福尼亚大学的 F. Stuart Chapin III 教 授利用开顶式同化箱对冻原草丛进行了长达 9 年的 系统研究之后, 得出植物和生态系统对增温的长、短 期反应不同的重要结论 (Chapin et al ., 1995) ; 美国 海洋生物实验室的 Jerry Melillo 等人利用加热电缆 方法对哈佛森林生态系统进行了 10 年的土壤加热 实验 (Melillo et al. 2002), 他们发现由于加热引起
的土壤呼吸的增加幅度随着时间推移逐渐降低, 这 主要是由于土壤中的活性碳逐渐消耗所致。Emmett 等(2004) 利用红外线反射器在欧洲进行的梯度实 验结果表明:土壤呼吸在增温条件下得以加强, 但不 同地点的土壤呼吸其温度敏感性 $\left(Q_{10}\right)$ 差别很大。 加利福尼亚大学 John Harte 教授利用红外线辐射器 对亚高山草甸生态系统进行了一系列的研究, 在 Science 上发表了重要研究结论即增温改变了植物 群落的物种组成 (Harte et al. , 1995) ; 俄克拉荷马大 学的 Yiqi Luo 研究组利用红外线辐射器对美国高草 草原进行了增温处理, 发现土壤呼吸的温度敏感性 (Temperature sensitivity) 在增温后降低, 导致陆地生 态系统在全球变暖后所释放的碳可能比预期的要少 (Luo et al. ,2001)。利用红外线辐射器取得重要学 术成果的还有 Weltzin 等 (2000) 对于沼泽生态系统 的研究以及 Zavaleta 等 (2003) 对于一年生植物占优 
势的加利福尼亚草地生态系统的研究。Weltzin 等 (2000) 认为不同物种和生活型对增温有着不同的反 应,Zavaleta等(2003)认为由增温引起的植物早衰现 象降低了植物蒸腾速率, 从而解释了增温条件下出 现的意想不到的土壤湿度增加的现象。通过上述事 例可以看出, 正是各个增温装置的不断改进和应用 推动了全球变化生态学研究的逐步深入发展。

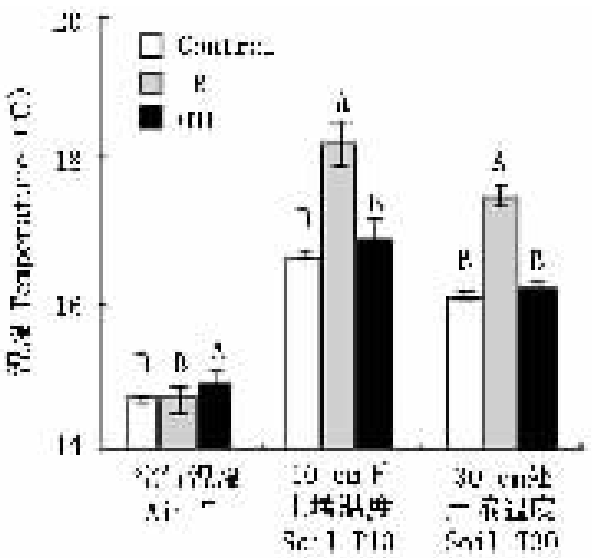

图 3 对照样地 (Control)、红外线辐射器加热样地 (IR)和开顶 箱加热样地 (OTC) 的空气温度以及 $10 \mathrm{~cm}$ 和 $30 \mathrm{~cm}$ 处 土壤温度季节均值的比较

Fig.3 Seasonal averages of air temperature, soil temperature at the depths of $10 \mathrm{~cm}$ and $30 \mathrm{~cm}$ in the control plots and warmed plots manipulated with

infrared radiator (IR) and open-top chambers (OTC)

不同的大写字母代表处理间差异显著性达到 0.05 水平 (数据来 自中国科学院植物研究所多伦全球变化多因子实验) Different capital letters represent statistically significant at $p<0.05$ (Data from Global Change Multi-factor Experiment in Duolun , Inner Mongolia sponsored by Institute of Botany , Chinese Academy of Sciences)

\section{3 研究前景及应注意的问题}

增温控制实验有助于解释生态系统响应和适应 全球气候变化的内在机制, 并为模型预测和验证提 供参数估计 (Dunne et al. , 2004)。但是现有的实验 方法仍然存在一些亟待解决的问题。

\section{1 增温装置之间的比较}

以前的增温实验都是在不同类型的生态系统中 运用不同的增温装置，使得增温实验结果的整合分 析存在困难 增加了模型预测的不确定性。如上所 述, 不同增温装置的加热机制不同, 可能会引起陆地 植物和生态系统特异性的反应。尽管 Rustad 等 (2001) 通过 Meta-analysis 的方法对土壤呼吸、土壤 N 矿化以及植物生长对增温的反应做了整合, 认为加 热装置对这些指标的影响不显著;但是这种整合是 将不同地点、不同植被类型的实验结果平均起来进 行比较的。正如 Rustad 所说的那样, 这些指标对温 度的反应程度在更大程度上依赖于实验地点和植被
类型(Shaver et al. , 2000) , 而不是增温幅度本身。 因此, 这种整合分析所得到的不同增温装置之间的 比较结果并不能真实地反映增温装置间的真实差 异。在同一个地点 (或生态系统) 同时设置多种增温 装置, 并比较其对陆地植物和生态系统影响的异同， 可以有效地整合不同生态系统增温实验的结果，完 善模型的参数估计和验证。

\section{2 实验的长期性}

生态系统不同组分和过程对温度的敏感性不 同 短期的增温实验往往不能得出作为整体的生态 系统响应与适应全球变暖的正确结论。例如运用开 顶箱在对 Tussock 苔原进行的加热实验中 植被生物 量在最初的 3 年对增温的反应是由实验小区内最初 出现的个体的生长反应所主导的, 这种短期的生物 量反应很难作为 9 年实验结果的预测 (Chapin et $a l$. , 1995)。同样, 在运用开顶箱对高海拔地区的加 热实验中，在最初的 3 年里植被的生长量都得到促 进，但是在第四年的实验中却出现了变化多样的结 果(Arft et al. , 1999) , 这是因为植物群落对于长期 的资源反馈、生长和竞争等效应有着滞后反应。长 期的增温反应可能被水分或养分等环境条件所限 制, 这使得植物或群落的长期与短期反应不同。在 美国 Harvard Forest 10 年的增温实验表明 增温对土 壤呼吸的促进作用随着时间的推移逐渐降低 (Melillo et al .,2002)。在 Rocky Mountain 亚高山草甸 ,在 增温的最初 10 年, 由于生产力较低的灌木逐渐替代 了生产力较高的非禾本科草本, 导致地下部分碳输 入和土壤有机碳含量逐渐降低; 然而模型预测结果 表明，由于灌木的凋落物质量会导致分解的减慢，最 终土壤有机碳含量降低的趋势会得到逆转 (Saleska et al .,2002)。

\section{3 梯度方法}

尽管野外增温实验是研究全球变化与陆地生态 系统关系的有效方法，但是由于控制实验侧重于生 态系统水平，在空间和时间尺度上均受到限制，在实 验结果和结论的外推 (Extrapolation) 和为区域和全球 水平的模型提供参数时仍然存在尺度上的问题 (Villalba et al . , 1994)。通过沿环境因子(如温度、 水分等)梯度的控制实验, 可以实现从当地(Local)到 区域 (Regional) 尺度的转换，同时以空间代替时间反 映植被和土壤对环境条件的长期反应结果, 完善不 同时空尺度模型之间的耦合。沿环境因子梯度进行 增温实验已受到越来越多的关注 (Saleska et al. , 
\& Wardle ,2005)。

\section{4 多因子控制实验}

全球变化涉及多项驱动因子 (大气层 $\mathrm{CO}_{2}$ 浓度、 温度、降水格局和土地利用) 的同步改变, 这些因子 之间可能存在复杂的相互作用。例如 $\mathrm{CO}_{2}$ 浓度升高 可以改变植物组织的化学组成和生态系统的水分平 衡这两者都能影响植物对温度的反应 (Shaw et $a l$. , 2002)。增温后植物的生长季节延长, 但生育期 同时也随着干旱期的延长而缩短; 另外氮的有效性 以及土地利用方式和人类干扰等因素都影响植物对 温度的反应程度。因此, 单一因子的控制实验并不 能真实地模拟全球变化对陆地植物和生态系统的潜 在影响; 在条件许可时, 开展多项因子的野外控制实 验将推动全球变化生态学的发展 (Shaw et al., 2002 ; Pendall et al. , 2004 , Norby \& Luo , 2004)。

全球变暖不仅能通过升温直接影响陆地植物和 生态系统, 而且可以通过改变土壤水分、凋落物分 解、土壤氮矿化和可利用性、物种组成、植物物候等 间接地影响陆地植物和生态系统 (Shaver et al. , 2000 ; Wan et al ，2005)。区分和量化全球变暖的 直接和间接效应, 将增加我们关于陆地植物和生态 系统对全球变暖的响应和适应机制的理解, 这也是 当前全球变化生态学需要解决的问题和面临的挑 战。

由于 $\mathrm{CO}_{2}$ 浓度对植物光合作用的限制, 多数模 型预测 :在全球变化背景下, 大气层中 $\mathrm{CO}_{2}$ 浓度的升 高将会增加陆地植被和生态系统的碳储量 ( IPCC , 2001)。但是这种预测是基于土壤氮供应充足的假 设之上的。如果考虑土壤中氮的可利用性对植物生 长的限制作用, 陆地植被所能固定的碳将远远低于 以前的预计 (Hungate et al. ,2003)。土壤中可利用 性氮含量取决于氮输入和输出之间的平衡。全球变 化 (包括 $\mathrm{CO}_{2}$ 浓度升高) 引起植物生长的增加, 将导 致更多的土壤有效氮被逐渐固定到植物生物量或土 壤有机物中; $\mathrm{CO}_{2}$ 浓度升高将降低植物凋落物的氮 含量, 引起调落物分解速率下降, 降低了氮素归还的 速率; 同时全球变化还可能导致生物固氮能力的下 降 (Hungate et al . ,2004); 述多方面因素作用的结 果 将会降低土壤中可利用性氮含量, 进而限制陆地 植物的生长和生态系统碳储量, 这种现象被称作渐 进式氮限制 (Progressive nitrogen limitation)。这种渐 进式氮限制假说于 2004 年提出之后 (Luo et al. , 2004 ），尽管科学家们已利用现有的实验结果对其进 行了检验( 见 2006 年 Ecology 87 卷第一期第 $3 \sim 75$
页的 Special Feature），但仍然缺乏有效的证据。

可以预计，对上述两个关键科学问题的探索将 促进全球变化生态学的发展。

\section{4 中国全球变暖的研究及增温装置的应用}

中国有着丰富的生态系统类型，主要陆地生态 系统的碳循环过程对于区域乃至全球的碳循环和平 衡起着重要的作用。因此, 研究全球变化条件下中 国主要陆地生态系统的响应与适应的生物学和生态 学机制对于理解我国的碳储量格局、碳循环过程和 源汇动态等问题具有重要的学术和实际指导意义。

我国科学家在全球变暖与陆地生态系统 (特别 是样带) (张新时和杨奠安, 1995;Peng \& Ren , 2000) 研究中做了大量卓有成效的工作, 然而这些工作大 部分是室内实验、野外调查和数据整合 (Fang et al. , 2001 ; Zhao \& Zhou，2005) 以及模型预测(张新时等， 1997 ; Gao \& Yu , 1998 ; Gao \& Zhang , 2000 ; Zhou et $a l$ ，2002；周广胜等，2003）,对于在野外自然条件 下生态系统尺度上进行的温度控制实验 (Ecosystem warming studies)投入精力较少。已知的两篇比较好 的研究报告 (Klein et al., 2004 ; Klein et al.,2005) 是由中国科学院西北高原生物研究所与美国加州大 学伯克利分校合作在青藏高原完成的。这项实验使 用的增温装置是开顶箱。如前所述, 开顶箱在白天 温度较高时增加日最高温度 (有时高达 $20{ }^{\circ} \mathrm{C}$ ) 会给 植物造成高温胁迫 (Klein et al ，2004; Musil et al.， 2004），这种白天高温胁迫所引起的陆地植物和生态 系统的反应与全球变暖背景下增温幅度白天低而夜 间高所引起的反应可能完全不同, 因此其实验结果 并不能外延到全球变暖背景下对本地和其它地区的 生态系统进行中长期预测。

2004 年 5 月,由中国科学院植物研究所植物生 态学研究中心发起的一项包括割草、施肥、浇水和增 温 4 种因子的全球变化多因子控制实验在内蒙古多 伦县展开, 以研究气候变化和人类活动对于我国典 型草原和农牧交错区生态系统的潜在影响。该实验 是目前国内第一个、也是规模最大的野外生态学控 制实验，该实验首次在国内应用红外线辐射器进行 增温处理。2006 年, 在国家自然科学基金委的资助 下, 一项在我国温带典型草原研究 全球变暖背景下 白天和夜间增温对温带草原群落结构和生态系统功 能的特异性影响” 的增温实验和一项沿降水梯度分 别在我国北方温带草甸草原、典型草原和荒漠化草 原进行增温和氮素处理的样带控制实验已全面启 


\section{动。上述这些温度控制实验的开展 , 将会增加我们 关于温带草原响应和适应全球变化格局和机制的理 解,为我国陆地生态系统的群落结构、初级生产力和 碳储量的中长期动态模型预测和检验提供可靠的参 数, 为我国在相关的国际谈判中提供必要的基础科 学数据。}

\section{参 考 文 献}

Arft AM, Walker MD, Gurevitch J, Alatalo JM, Bret-Harte MS, Dale M, Diemer M, Gugerli F, Henry GHR, Jones MH, Hollister RD, Jónsdóttir IS, Laine K, Lévesque E, Marion GM, Molau U, Mфlgaard P, Nordenhäll U, Raszhivin V, Robinson CH, Starr G, Stenström A, Stenström M, Totland $\phi$, Turner PL, Walker LJ, Webber PJ, Welker JM, Wookey PA (1999). Response patterns of tundra plant species to experimental warming: a meta-analysis of the International Tundra Experiment. Ecological Monographs, 69, 491-512.

Beier C, Emmett B, Gundersen P, Tietema A, Penuelas J, Estiarte M, Gordon C, Gorissen A, Llorens L, Roda F, Williams D (2004) . Novel approaches to study climate change effects on terrestrial ecosystems in the field: drought and passive nighttime warming. Ecosystems, 7, 583 - 597.

Bergh J, Linder S (1999). Effects of soil warming during spring on photosynthetic recovery in boreal Norway spruce stands. Global Change Biology, 5, 245-253.

Bridgham SD, Pastor J, Updegraff K, Janssens JA, Malterer TJ (1995). Paper presented at the Ecological Society of America Annual Meeting, Snowbird, Utah, July 30 to August 3.

Chapin FS, Shaver GR, Giblin AE, Nadelhoffer KJ, Laundre JA (1995) . Responses of arctic tundra to experimental and observed changes in climate. Ecology, 76, 694-711.

Chapin PS III, Shaver GR (1985). Individualistic grow response of tundra plant species to environmental manipulations in the field. Ecology, 66, 564 - 576.

Day TA, Ruhland CT, Grobe CW, Xiong F (1999). Growth and resproduction of Antarctic vascular plants in response to warming and UV radiation reduction in the field. Oecologia, 119, $24-$ 35 .

Debevec EM, Maclean SF (1993). Design of greenhouses for the manipulation of temperature in tundra plant communities. Arctic Antarctic and Alpine Research, 25, 56-62.

Dunne JA, Saleska SR, Fischer ML, Harte J (2004) . Integrating experimental and gradient methods in ecological climate change research. Ecology, 85, 904-916.

Emmett BA, Beier C, Estiarte M, Tietema A, Kristensen HL, Williams D, Peñuelas J, Schmidt I, Sowerby1 A (2004). The response of soil processes to climate change: results from manipulation studies of shrublands across an environmental gradient. Ecosystems, 7, $625-637$.

Fang J, Chen A, Peng C, Zhao S, Ci L (2001). Changes in forest biomass carbon storage in China between 1949 and 1998. Science, 292, $2320-2322$.

Fukami T, Wardle DA (2005) . Long-term ecological dynamics: reciprocal insights from natural and anthropogenic gradients. Proceedings of the Royal Society of London Series B: Biology Sciences, $272,2105-2115$.

Gao Q, Yu M (1998). A model of regional vegetation dynamics and its application to the study of the Northeast China Transect (NECT) responses to global change. Global Biogeochemical Cycles, $12,329-344$.

Gao Q, Zhang XA (2000). A simulation study of the responses of the northeast China Transect to elevated $\mathrm{CO}_{2}$ and climate change. Ecological Applications, 7, 470-483.

Harte J, Torn MS, Chang FR, Feifarek B, Kinzig, AP, Shaw R, SHen K (1995). Global warming and soil microclimate: results from a meadow-warming experiment. Ecological Applications, 5, $132-150$.

Hartley AE, Neill C, Melillo JM, Crabtree R, Bowles FP (1999). Plant performance and soil nitrogen mineralization in response to simulated climate change in subarctic dwarf heath. Oikos, 86, $331-343$.

Havström M, Challaghan TV, Jonasson S ( 1993 ). Differential growth responses of Cassiope tetragona, an arctic dwarf-shrub, to environmental perturbations among three contrasting high and subarctic sites. Oikos, 66, 389-402.

Hiller SH, Sutton F, Grime JP (1994). A new technique for the experimental manipulation of temperature in plant communities. Functional Ecology, 8, 755 - 762 .

Hobbie SE, Shevtsova A, Chapin PS III (1999). Plant response to species removal and experimental warming in Alaskan tussock tundra. Oikos, 84, 417-434.

Hollister RD, Webber PJ (2000). Biotic validation of small opentop chambers in a tundra ecosystem. Global Change Biology, 6, $835-842$.

Hungate BA, Dukes JS, Shaw MR, Luo Y, Field CB (2003). Nitrogen and climate change. Science, 302, $1512-1513$.

Hungate BA, Stiling PD, Dijkstra P, Johnson DW, Ketterer ME, Hymus GJ, Hinkle CR, Drake BG (2004). $\mathrm{CO}_{2}$ elicits long-term decline in nitrogen fixation. Science, 304, 1291.

Ineson P, Benham DG, Poskitt J, Harrison AF, Taylor W, Woods C (1998). Effects of climate change on nitrogen dynamics in upland soils. II. A soil warming study. Global Change Biology, 4, $153-162$.

IPCC (2001). Climate change 2001: the scientific basis: summary for policymakers. IPCC WGI Third Assessment Report. Shanghai Draft, 21 January 2001.

Kennedy AD (1995). Simulated climate change: are passive greenhouses a valid microcosm for testing the biological effects of environmental perturbations? Global Change Biology, 1, 29-42.

Kimball BA (2005). Theory and performance of an infrared heater for warming ecosystems. Global Change Biology, 11, 2041 - 
2056.

Klein JA, Harte J, Zhao XQ (2004) . Experimental warming causes large and rapid species loss, dampened by simulated grazing, on the Tibetan Plateau. Ecology Letters, 7, 1170 - 1179.

Klein JA, Harte J, Zhao XQ (2005) . Dynamic and complex microclimate responses to warming and grazing manipulations. Global Change Biology, 11, 1440 - 1451 .

Luo Y, Su B, Currie WS, Dukes JS, Finzi A, Hartwig U, Hungate B, McMurtrie RE, Oren R, Parton WJ, Partaki DE, Shaw MR, Zak DR, Field CB (2004). Progressive nitrogen limitation of ecosystem responses to rising atmospheric carbon dioxide. BioScience, 54, $731-739$.

Luo Y, Wan S, Hui D, Wallace LL (2001). Acclimatization of soil respiration to warming in tallgrass prairie. Nature, 413, $622-$ 625 .

Luxmoore RJ, Hanson PJ, Beauchamp JJ, Joslin JD (1998) . Passive nighttime warming facility for forest ecosystems research. Tree Physiology, 18, 615-623.

Marion GM, Henry GHR, Freckman DW, Johnstone J, Jones G, Jones MH, Levesque E, Molau U, Molgaard P, Parsons AN, Svoboda J, Virginia RA (1997). Open-top designs for manipulating field temperature in high-latitude ecosystems. Global Change Biology, 3 (Suppl. 1), 20-32.

Melillo JM, Steudler PA, Aber JD, Newkirk K, Lux H, Bowles FP, Catricala C, Magill A, Ahrens T, Morrisseau S (2002). Soil warming and carbon-cycle feedbacks to the climate systems. Science, 298, $2173-2176$.

Musil CF, Schmidel U, Midgley GF (2004). Lethal effects of experimental warming approximating a future climate scenario on southern African quartz-field succulents: a pilot study. New Phytologist, 165, $539-547$.

Nijs I, Kockelbergh F, Teughels H, Blum H, Hendrey G, Impens I (1996) . Free Air Temperature Increase (FATI) : a new tool to study global warming effects on plants in the field. Plant, Cell, and Environment, 19, 495-502.

Noormets A, Chen J, Bridgham S, Weltzin JF, Pastor J, Dewey B, LeMoine J (2004). The effects of infrared loading and water table on soil energy fluxes in northern peatlands. Ecosystems, 7, 573 -582 .

Norby RJ, Edwards NT, Riggs JS, Abner CH, Wullschleger SD, Gunderson CA (1997). Temperature-controlled open-top chambers for global change research. Global Change Biology, 3, 259 -267 .

Norby RJ, Luo Y (2004) Evaluating ecosystem responses to rising atmospheric $\mathrm{CO}_{2}$ and global warming in a multi-factor world. New Phytologist, 162, $281-293$.

NSFESP ( National Science Foundation, Ecosystem Studies Program) (1991). Soil-warming experiments in global change research, Woods Hole, MA, September 27 and 28.

Oechel WC, Vourlitis GL, Hastings SJ, Ault RP, JrBryant P (1998). The effects of water table manipulation and elevated temperature on the net $\mathrm{CO}_{2}$ flux of wet sedge tundra ecosystems. Global Change Biology, 4, 77 - 90 .

Pendall E, Bridgham S, Hanson PJ, Hungate B, Kicklighter DW, Johnson DW, Law BE, Luo Y, Megonigal JP, Olsrud M, Ryan MG, Wan S (2004). Below-ground process response to elevated $\mathrm{CO}_{2}$ and temperature: a discussion of observations, measurement methods, and models. New Phytologist, 162, $311-322$.

Peng SL, Ren H (2000). The North-South Transect of Eastern China (NSTEC) for global change studies. GCTE News, 16, 6.

Peterjohn WT, Melillo JM, Bowles FP, Steudler PA (1993). Soil warming and trace gas fluxes: experimental design and preliminary flux results. Oecologia, 93, $18-24$.

Peterjohn WT, Melillo JM, Steudler PA, Newkirk KM, Steudler PA, Newkirk KM, Bowles FP, Aber JD (1994). Responses of trace gas fluxes and $\mathrm{N}$ availability to experimentally elevated soil temperatures. Ecological Applications, 4, 617-625.

Richardson SJ, Hartley SE, Press MC (2000). Climate warming experiments, are tens a potential barrier to interpretation? Ecological Entomology, 25, 367 - 370 .

Robinson CH, Woodey PA, Lee JA, Callaghan TV, Press MC (1998). Plant community responses to simulated environmental change at a high Arctic polar semi-desert. Ecology, 79, 856866 .

Rustad LE, Campbell JL, Marion GM, Norby RJ, Mitchell MJ, Hartley AE, Cornelissen JHC, Gurevitch J, GCTE-NEWS (2001) . A meta-analysis of the response of soil respiration, net nitrogen mineralization, and aboveground plant growth to experimental ecosystem warming. Oecologia, 126, $543-562$.

Rykbost KA, Boersma L, Mack HJ. Schmisseur WE (1975). Yield response to soil warming: agronomic crops. Agronomy Journal, $67,733-738$.

Saleska SR, Shaw MB, Fischer ML, Dunne JA, Still CJ, Holman ML, Harte J (2002). Plant community composition mediates both large transient decline and predicted long-term recovery of soil carbon under climate warming. Global Biogeochemical Cycles, 16, 1055, doi:10.1029/2001GB1573.

Shaver GR, Canadell J, Chapin III FS, Gurevitch J, Harte J, Henry G, Ineson P, Jonasson S, Melillo J, Pitelka L, Rustad L (2000). Global warming and terrestrial ecosystems: a conceptual framework for analysis. BioScience, 50, 871-882.

Shaver GR, Johnson LC, Cades DH, Murray G, Laundre JA, Rastetter EB, Nadelhoffer KJ, Giblin AE (1998). Biomass and $\mathrm{CO}_{2}$ flux in wet sedge tundra, response to nutrients, temperature, and light. Ecological Monographs, 68, 75 - 99.

Shaw MR, Zavaleta ES, Chiariello NR, Cleland EE, Mooney HA, Field CB (2002). Grassland responses to global environmental changes suppressed by elevated $\mathrm{CO}_{2}$. Science, 298, 1987 1990 .

Shen KP, Harte J (2000). Ecosystem climate manipulations. In: Sala OE, Jackson RB, Mooney HA, Howarth RW eds. Methods in Ecosystem Science. Springer-Verlag Press, New York, 353 - 
369.

Stenstrom M, Gugerli F, Henry GHR (1997). Response of Saifraga oppositifolia $\mathrm{L}$. to simulated climate change at three contrasting latitudes. Global Change Biology, 3 (Suppl. 1), 44-54.

Suzuki S, Kudo G (1997). Short-term effects of simulated environmental change on phenology, leaf traits, and shoot growth of alpine plants on a temperate mountain, northern Japan. Global Change Biology, 3 (Suppl.1), $108-115$.

van Cleve K, Dyrness CT, Viereck LA, Fox J, Chapin FS, Oeschel W (1983). Tiaga ecosystems in interior Alaska. BioScience, 33, 39-44.

van Cleve K, Oechel WC, Hom JL (1990). Response of black spruce ( Picea mariana) ecosystem to soil temperature modification in interior Alaska. Canadian Journal of Forest Research, 20 , $1530-1535$.

Verberg PSJ, Van Loon WKP, Lukewille A (1999). The climax soil-heating experiment: soil response after 2 years of treatment. Biology and Fertility of Soils, 28, $271-276$.

Villalba R, Veblen T, Ogden J (1994). Climatic influences on the growth of subalpine trees in the Colorado front range. Ecology, $75,721-733$.

Walker MD, Wahren CH, Hollister RD, Henry GHR, Ahlquist LE, Alatalo JM, Bret-Harte MS, Calef MP, Callaghan TV, Carroll AB, Epstein HE, Jónsdóttir IS, Klein JA, Magnússon B, Molau U, Oberbauer SF, Rewa SP, Robinson CH, Shaver GR, Suding KN, Thompson CC, Tolvanen A, Totland $\varnothing$, Turner PL, Tweedie CE, Webber PJ, Wookey PA (2006). Plant community response to experimental warming across the tundra biome. Proceedings of the National Academy of Sciences, 103, 1342 - 1346.

Wan S, Luo Y, Wallace LL (2002a). Changes in microclimate induced by experimental warming and clipping in tallgrass prairie. Global Change Biology, 8, 754 - 768 .

Wan S, Yuan T, Bowdish S, Wallace L, Russell SD, Luo Y $(2002 \mathrm{~b})$. Response of an allergic species, Ambrosia psilostochya, to experimental warming and clipping: implications for public health under global change. American Journal of Botany, 89, 1843 - 1846.

Wan SQ, Hui DF, Wallace L, Luo YQ (2005). Direct and indirect effects of experimental warming on ecosystem carbon processes in a tallgrass prairie. Global Biogeochemical Cycles, 19, GB2014, doi : 10. 1029/2004GB002315.

Weltzin JF, Pastor J, Harth C, Bridgham SD, Updegraff K, Chapin CT (2000) . Response of bog and fen plant communities to warming and water-table manipulations. Ecology, 81, 3464-3478.

Werkman BR, Callaghan TV, Welker JM (1996). Responses of bracken to increased temperature and nitrogen availability. Global Change Biology, 2, 59-66.

Zavaleta ES, Thomas BD, Chiariello NR, Asner GP, Shaw MR, Field CB (2003). Plants reverse warming effect on ecosystem water balance. Proceedings of the National Academy of Sciences, $100,9892-9893$.

Zeiher CA, Brown PW, Silvertooth JC, Matumba N, Milton N (1994). The effect of night temperature on cotton reproductive development. In: Silvertooth J ed. Cotton. College of Agriculture Report, the University of Arizona, Tucson USA. 89-96.

Zhang XS (张新时), Gao Q(高琼), Yang DA(杨殿安), Zhou GS (周广胜), Ni J(倪健), Wang Q(王权) (1997). A gradient analysis and prediction on the northeast China transect (NECT) for global change study. Acta Botanica Sinica (植物学报), 39, 785 - 799. (in Chinese with English abstract)

Zhang XS(张新时), Yang DA(杨奠安) (1995). Allocation and study on global change transects in China. Quaternary Sciences (第四纪研究) , 1, 43-52. (in Chinese with English abstract)

Zhao M, Zhou G (2005). Estimation of biomass and net primary productivity of major forest planted forests in China based on forest inventory data. Forest Ecology and Management, 207, 295 313.

Zhou G, Wang Y, Wang S (2002). Responses of grassland ecosystems to precipitation and land use along the Northeast China Transect. Journal of Vegetation Science, 13, 361 - 368 .

Zhou GS(周广胜), Wang YH(王玉辉), Xu ZZ(许振柱), Zhou L (周莉), Jiang YL(蒋延玲) (2003). Advances of study on carbon cycles on the northeast China transect (NECT). Progress in Natural Science (自然科学进展), 13,917-922. (in Chinese) 